\title{
Grosores Tisulares Faciales en Cadáveres de Españoles y su Aplicación en la Identificación Médicolegal
}

\author{
Tissue Tickness in Spanish Corpses and your Applications on the Medicolegal Identification \\ Iván Claudio Suazo Galdames; Francisco Javier Pérez Rojas \& Sebastián René Torres Muñoz
}

SUAZO, G. I. C., PÉREZ, R. F. J. \& TORRES, M. S. R. Grosores tisulares faciales en cadáveres de españoles y su aplicación en la identificación médicolegal. Int. J. Morphol., 25(1):109-116, 2007.

RESUMEN: La reconstrucción facial a través de la escultura forense es una técnica en la cual, mediante el modelado con materiales plásticos sobre un esqueleto cefálico, se reproducen la estructuras blandas de la cara, con el propósito de ayudar al proceso de identificación médicolegal. Uno de los datos más relevantes para reproducir esta técnica corresponde a los valores de grosor tisular en determinados puntos del esqueleto craneal.

En el presente estudio se analizaron los grosores tisulares, en 14 puntos craneométricos medianos y paramedianos de 32 cadáveres de individuos, españoles mediante la introducción de agujas en la piel. Los cadáveres fueron divididos en grupos de acuerdo a sexo y grupo etáreo.

Se encontraron diferencias significativas en los valores de grosor tisular entre hombres y mujeres de ambos grupos etáreos, observándose en los hombres un mayor grosor tisular en los puntos medianos, mientras que en las mujeres resultaron mayores los grosores medidos en puntos paramedianos.

PALABRAS CLAVE: Grosor tisular; Antropología forense; Identificación humana.

\section{INTRODUCCIÓN}

La reconstrucción facial por escultura forense es un procedimiento mediante el cual se aplica un material moldeable sobre una cabeza ósea, con el fin de reproducir de manera inferencial el aspecto que tendría el sujeto (Teke, 2004). Esta técnica se debe realizar una vez que se ha logrado estimar la raza, edad, sexo y talla del individuo al cual perteneció la osamenta (Farkas, 1994).

La reconstrucción mediante escultura forense está indicada cuando no se encuentran evidencias para la identificación de la osamenta. La técnica consiste en colocar capas sucesivas de un material plástico y moldeable, como la arcilla, utilizando como referencias una serie de puntos anatómicos y antropométricos, sobre los cuales se colocan topes de madera o plástico cuyo grosor determina el grosor tisular del segmento que se está modelando (Rodríguez, 2003).

Si bien la técnica de escultura forense es descrita de manera similar por numerosos autores, el grosor que deben tener los topes de referencia presenta diferencias (Wilkinson, 2002).
En individuos vivos, se ha descrito que el grosor tisular se encuentra influenciado por factores como la edad y el sexo del paciente (El-Mehallawi \& Soliman, 2001). En cadáveres, estas medidas se relacionan también con los procesos de deshidratación y presentan variaciones dependiendo del medio de conservación.

En el presente estudio se analizan los grosores del tegumento facial en distintos puntos de la cabeza y se establecen correlaciones con sexo y edad.

\section{MATERIAL Y MÉTODO}

Fueron estudiados 32 cadáveres de individuos españoles, obtenidos del Laboratorio de Anatomía Humana de la Universidad Cardenal Herrera de Valencia, España, conservados mediante la técnica de inyección intravascular de solución fijadora conservadora en base a formaldehido, que presentaban un índice de masa corporal normal (18,5 a 24.9 $\mathrm{Kg} / \mathrm{m}^{2}$ ), sin cicatrices ni deformidades faciales. 
La muestra estuvo integrada por 32 cadáveres, 16 entre 24 y 35 años de edad ( 8 mujeres y 8 hombres) y 16 entre 36 y 50 años de edad ( 8 mujeres y 8 hombres).

En cada cadáver se identificaron y marcaron 14 puntos cefalométricos, de los cuales 8 se encontraron en la línea mediana y 6 fueron paramedianos, bilaterales (Fig. 1; Tabla I).

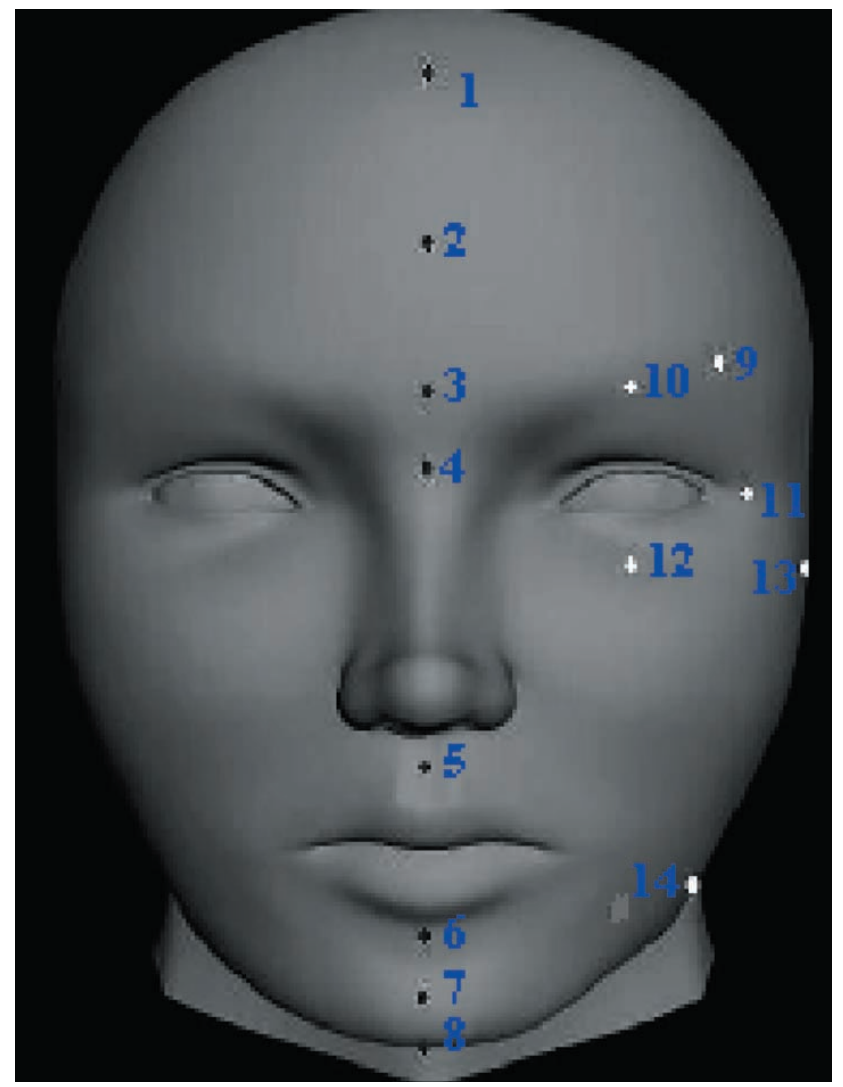

Fig. 1. Puntos cefalométricos medianos y paramedianos utilizados en el estudio de grosres tisulares. (Lectura de números en Tabla I).

En cada punto se realizó la medición del grosor del tegumento por medio de la inserción de una aguja envaselinada de $40 \mathrm{~mm}$ de largo y 27 Gauge, que presentaba un tope de goma. La inserción se realizó de manera perpendicular a la superficie cutánea, evitando la formación de pliegues, profundizando hasta encontrar contacto óseo (Fig.2).
Se midió la distancia entre la punta de la aguja y el comienzo del tope de goma, utilizando una regla milimetrada.

Los resultados obtenidos fueron tabulados y se realizaron correlaciones de los grosores con el sexo y la edad de los cadáveres, se aplicaron estadísticas no paramétricas para analizar la significancia de esas correlaciones.

\section{RESULTADOS}

En la Tabla II se presentan los valores y la correlación existente entre el grosor tisular medido en puntos medianos y paramedianos en cadáveres de sexo masculino. Los resultados muestran diferencias significativas $(p>0.05)$ al comparar los dos grupos etáreos, en los puntos B de Downs, gnathion, supraorbitario, infraorbitario, zygion, trichion, A de Downs, pogonion, exocanthion y gonion.

En la Tabla III figuran los valores y la correlación existente entre el grosor tisular medido en puntos medianos y paramedianos en cadáveres de sexo femenino. Los resultados muestran diferencias significativas $(p>0.05)$, al comparar los dos grupos etáreos, en los puntos trichion, glabela, A de Downs, pogonion, infraorbitario, zygion, gonion, supraglabela, nasion, B de Downs, gnathion, supraorbirario y exocanthion.

En la Tabla IV se muestran los valores de grosor tisular medidos en puntos medianos y paramedianos del grupo etáreo entre 24 y 35 años y las correlaciones existentes de acuerdo al sexo. Los resultados muestran diferencias significativas ( $p>0.05)$, al comparar cadáveres masculinos $\mathrm{y}$ femeninos, en los puntos nasion, trichion, supraglabela, A de Downs, B de Downs, gnathion, Zygion y gonion.

La Tabla V muestra los valores de grosor tisular, medidos en puntos medianos y paramedianos del grupo etáreo entre 36 y 50 años y las correlaciones existentes de acuerdo al sexo. Los resultados muestran diferencias significativas $(p>0.05)$, al comparar cadáveres masculinos y femeninos, en los puntos supraglabela, glabela, A de Downs, B de Downs, pogonion, supraorbitario, infraorbitario, zygion, nasion, superciliar y gonion.

Fig. 2. Fotografías de las mediciones en el tercio superior (A) y en el tercio inferior de la cara (B). Obsérvese el tope de goma y la ausencia de tensión en el tejido. 
Tabla I. Puntos cefalométricos medianos y paramedianos utilizados en el estudio de los grosores tisulares faciales en cadáveres y su aplicación en la identificación médicolegal.

\begin{tabular}{|c|c|}
\hline Trichion & $\begin{array}{l}\text { Punto ubicado en la línea mediana en la zona del nacimiento del pelo (como es variable se elige una medida a } 6 \\
\mathrm{~cm} \text { de la glabela). }\end{array}$ \\
\hline Supraglabela & Punto ubicado en la línea mediana a $2 \mathrm{~cm}$ sobre la glabela. \\
\hline Glabela & $\begin{array}{l}\text { Punto más prominente sobre el seno frontal, situado entre las cejas en la línea mediana e idéntico a la glabela en } \\
\text { el hueso frontal. }\end{array}$ \\
\hline Nasion & $\begin{array}{l}\text { Representa la unión del hueso nasal con el hueso frontal, es decir, la sutura frontonasal en el punto más posterior } \\
\text { de la curvatura en el puente de la nariz, que tiene una representación en el tejido blando en el punto más cóncavo } \\
\text { retruido que cubre el área de la sutura frontonasal. }\end{array}$ \\
\hline A de Downs & Punto más anterior sobre el margen del labio membranoso superior. \\
\hline B de Downs & Punto más inferior sobre el margen del labio membranoso inferior. \\
\hline Pogonion & $\begin{array}{l}\text { Punto más anterior de la línea mediana mandibular. En el tejido blando corresponde al punto más prominente o } \\
\text { anterior del tejido blando del mentón en el plano mediano. }\end{array}$ \\
\hline Gnathion & $\begin{array}{l}\text { Punto más superior que se encuentra más hacia delante de la curvatura que se observa de perfil de la línea } \\
\text { mediana mandibular. En el tejido blando, se encuentra entre el punto anterior y el inferior del mentón en el plano } \\
\text { mediano. }\end{array}$ \\
\hline Supraciliar & Punto lateral situado en la parte más prominente del hueso frontal. \\
\hline Supraorbitario & Punto más alto de la órbita ósea. \\
\hline Exocanthion & Punto situado en la comisura lateral de la cavidad orbitaria. \\
\hline Infraorbitario & Punto más bajo en el margen inferior de cada órbita. \\
\hline Zygion & Punto más lateral del hueso cigomático, visto desde frente. \\
\hline Gonion & $\begin{array}{l}\text { Punto más superior y saliente del ángulo formado por la unión de la rama y el cuerpo de la mandíbula en su } \\
\text { región posteroinferior. }\end{array}$ \\
\hline
\end{tabular}

\section{DISCUSIÓN}

El conocimiento del grosor tisular permite al escultor forense estimar el volumen que debe modelar sobre la superficie craneal, teniendo como referencia determinados puntos cefalométricos.

El análisis y la correlación de los grosores del tegumento facial en distintos puntos de la cabeza, reveló la existencia de diferencias significativas en los hombres de ambos grupos etáreos estudiados. Se observó que los varones presentaban un mayor grosor de tejido blando en la zona correspondiente a la línea mediana facial, siendo máxima esta diferencia en la región nasal y en el mentón. Las mujeres, en cambio, presentaron un mayor grosor del tejido blando en ubicación paramediana, específicamente, en la zona correspondiente a la mejilla, lo que puede deberse a una mayor cantidad de tejido adiposo de ubicación superficial a la musculatura facial (Wilkinson). Estos datos corroboran las investigaciones descritas por diversos autores, tales como, Rhine \& Campbell, (1980); Dumont (1986) y Manhein et al. (2000), quienes han obtenido resultados similares. Las diferencias entre géneros se presentan desde la niñez y se acentúan durante la adolescencia, debido a que en la pubertad se llevan a cabo todos los procesos de diferenciación de caracteres sexuales, dentro de los cuales el volumen de los tejidos blandos del rostro está presente (Wilkinson).

En el adulto joven, las diferencias de la profundidad del tejido blando cráneo facial gestadas durante la pubertad, se mantienen estables (Simpson \& Henneberg, 2002) coincidiendo con los resultados obtenidos por este estudio. Sin embargo, otros estudios no encontraron variaciones significativas en adultos mayores de ambos sexos (Domaracki \& Stephan, 2006), existiendo zonas de mayor grosor de tejido, tanto en el hombre como en la mujer, pero que no proporcionan variabilidad alguna al momento de realizar una aproximación facial. Debido a lo anterior, resulta importante contar con una base de datos de grosores de tejido blando craneofacial, acorde a los diversos rangos etáreos existentes, con la finalidad de lograr una reproducción facial lo más exacta posible, de acuerdo a las características propias del individuo encontrado. 
Tabla II. Valores y correlación de los grosores tisulares medidos entre puntos medianos y paramedianos de 16 cadáveres de sexo masculino en ambos grupos etáreos.

\begin{tabular}{|c|c|c|c|c|c|c|c|}
\hline Puntos cefalométricos & $\begin{array}{l}\text { Grupo etáreo } \\
\text { (años) }\end{array}$ & Promedio & $\begin{array}{c}\text { Desviación } \\
\text { estándar }\end{array}$ & $\begin{array}{l}\text { U de Mann- } \\
\text { Whitney }\end{array}$ & $\mathrm{Z}$ & $\begin{array}{l}\text { Sig, sintótica } \\
\text { (bilateral) }\end{array}$ & $\begin{array}{l}\text { Sig. exacta } \\
\text { (unil ateral) }\end{array}$ \\
\hline \multirow[t]{2}{*}{ Trichion (1) } & 24 a 35 & 5,188 & 0,1885 & \multirow[b]{2}{*}{8,500} & \multirow[b]{2}{*}{$-2,484$} & \multirow[b]{2}{*}{0,013} & \multirow[b]{2}{*}{$0,010^{\mathrm{a}}$} \\
\hline & 36 a 50 & 4,750 & 0,5014 & & & & \\
\hline \multirow[t]{2}{*}{ Supraglabela (2) } & 24 a 35 & 4,675 & 0,1282 & \multirow[b]{2}{*}{18,500} & \multirow[b]{2}{*}{$-1,432$} & \multirow[b]{2}{*}{0.152} & \multirow[b]{2}{*}{$0,161^{\mathrm{a}}$} \\
\hline & 36 a 50 & 4,425 & 0,5175 & & & & \\
\hline \multirow[t]{2}{*}{ Glabela (3) } & 24 a 35 & 5,550 & 0,1604 & \multirow{2}{*}{16,500} & \multirow{2}{*}{$-1,634$} & \multirow{2}{*}{0.102} & \multirow{2}{*}{$0,105^{\mathrm{a}}$} \\
\hline & 36 a 50 & 5,325 & 0,8515 & & & & \\
\hline \multirow[t]{2}{*}{ Nasion (4) } & 24 a 35 & 5,988 & 0,1458 & \multirow{2}{*}{16,500} & \multirow{2}{*}{$-1,649$} & \multirow{2}{*}{0,099} & \multirow{2}{*}{$0,105^{\mathrm{a}}$} \\
\hline & 36 a 50 & 6,113 & 0,4518 & & & & \\
\hline \multirow[t]{2}{*}{ A de Downs (5) } & 24 a 35 & 11,250 & 0,1309 & \multirow{2}{*}{8,000} & \multirow{2}{*}{$-2,534$} & \multirow{2}{*}{0,011} & \multirow{2}{*}{$0,010^{\mathrm{a}}$} \\
\hline & 36 a 50 & 11,950 & 0,9040 & & & & \\
\hline B de Downs (6) & 24 a 35 & 10,238 & 0,1408 & & & & $0000^{\mathrm{a}}$ \\
\hline & 36 a 50 & 11,200 & 0,3338 & 0,000 & $-3,3 / 3$ & 0,001 & 0,000 \\
\hline Pogonion (7) & 24 a 35 & 11,675 & 0,1035 & & & & \\
\hline & 36 a 50 & 11,050 & 0,6188 & 11,500 & $-2,179$ & 0,029 & $0,028^{\mathrm{a}}$ \\
\hline Gnathion (8) & 24 a 35 & 6,675 & 0,0886 & & & & \\
\hline & 36 a 50 & 8,928 & 1,5583 & 0,000 & $-3,391$ & 0,001 & $0,000^{\mathrm{a}}$ \\
\hline S uperciliar izquierdo (9) & 24 a 35 & 5,838 & 0,1685 & 19,000 & $-1,136$ & 0,169 & $0,195^{\mathrm{a}}$ \\
\hline & 36 a 50 & 5,788 & 0,7396 & & & & \\
\hline Superciliar derecho (10) & 24 a 35 & 5,775 & 0,1389 & & & & \\
\hline & 36 a 50 & 5,850 & 0,5210 & 13,500 & $-1,990$ & 0,047 & $0,050^{a}$ \\
\hline Supraorbitario izquierdo (11) & 24 a 35 & 5,738 & 0,0744 & & & & \\
\hline & 36 a 50 & 7,975 & 1,0607 & 7,500 & $-2,608$ & 0,009 & $0,007^{\mathrm{a}}$ \\
\hline Supraorbitario derecho (12) & 24 a 35 & 5,875 & 0,0886 & & & & \\
\hline & 36 a 50 & 8,288 & 0,5167 & 0,000 & $-3,422$ & 0,001 & $0,000^{\mathrm{a}}$ \\
\hline Exocanthion izquierdo (13) & 24 a 35 & 3,725 & 0,1488 & & & & \\
\hline & 36 a 50 & 4,200 & 0,5318 & 8,500 & $-2,486$ & 0,013 & $0,010^{\mathrm{at}}$ \\
\hline Exocanthion derecho (14) & 24 a 35 & 3,763 & 0,1923 & & & & \\
\hline & 36 a 50 & 4,238 & 0,4596 & 9,00 & $-2,433$ & 0,015 & $0,015^{\mathrm{a}}$ \\
\hline Infraorbitario izquierdo (15) & 24 a 35 & 4,400 & 0,1773 & & & & \\
\hline & 36 a 50 & 7,213 & 0,7643 & 0,000 & $-3,386$ & 0,001 & $0,000^{2}$ \\
\hline Infraorbitario derecho (16) & 24 a 35 & 4,400 & 0,2673 & & & & \\
\hline & 36 a 50 & 7,088 & 0,5139 & 0,000 & $-3,373$ & 0,001 & $0,000^{\circ}$ \\
\hline Zygion izquierdo (17) & 24 a 35 & 5,000 & 0,2000 & & & & \\
\hline & 36 a 50 & 10,013 & 2,4701 & 8,000 & $-2,535$ & 0,011 & $0,010^{\mathrm{a}}$ \\
\hline Zygion derecho (18) & 24 a 35 & 4,988 & 0,2167 & & & & $0,000^{\mathrm{a}}$ \\
\hline & 36 a 50 & 10,225 & 1,9962 & 0,000 & $-3,388$ & 0,001 & \\
\hline Gonion izquierdo (19) & 24 a 35 & 5,163 & 0,1061 & & & & \\
\hline & 36 a 50 & 9,850 & 2,0935 & 7,500 & $-2,588$ & 0,010 & $0,007^{a}$ \\
\hline Gonion derecho (20) & 24 a 35 & 5,125 & 0,1581 & & & & \\
\hline & 36 a 50 & 9,750 & 1,9228 & 2,500 & $-3,135$ & 0,002 & $0,001^{\mathrm{a}}$ \\
\hline
\end{tabular}

a. No corregidos para los empates. b. Variable de agrupación. c. Sexo = masculino 
Tabla III. Valores y correlación de grosores tisulares medidos en pntos medianos y paramedianos de 16 cadáveres de sexo femenino en ambos grupos etáreos.

\begin{tabular}{|c|c|c|c|c|c|c|c|}
\hline Punto & $\begin{array}{l}\text { Grupo etáreo } \\
\text { años }\end{array}$ & Promedio & $\begin{array}{c}\text { Desviación } \\
\text { estándar }\end{array}$ & $\begin{array}{l}\text { U de Mann- } \\
\text { Whitney }\end{array}$ & $\mathrm{Z}$ & $\begin{array}{l}\text { Sig. asintótica } \\
\text { (bilateral) }\end{array}$ & $\begin{array}{l}\text { Sig, exacta } \\
\text { (unilateral) }\end{array}$ \\
\hline \multirow[t]{2}{*}{ Trichion } & 24 a 35 & 3,525 & 0,2816 & \multirow[b]{2}{*}{0,000} & \multirow[b]{2}{*}{$-3,368$} & \multirow[b]{2}{*}{0,001} & \multirow[b]{2}{*}{$0,000^{\mathrm{a}}$} \\
\hline & 36 a 50 & 4,775 & 0,3845 & & & & \\
\hline \multirow[t]{2}{*}{ S upraglabela } & 24 a 35 & 3,475 & 0,3012 & \multirow[b]{2}{*}{8,500} & \multirow[b]{2}{*}{$-2,486$} & \multirow[b]{2}{*}{0.013} & \multirow[b]{2}{*}{$0,010^{\mathrm{a}}$} \\
\hline & 36 a 50 & 3,875 & 0,2375 & & & & \\
\hline \multirow[t]{2}{*}{ Glabela } & 24 a 35 & 5,650 & 0,2507 & \multirow{2}{*}{0,000} & \multirow{2}{*}{$-3,373$} & \multirow{2}{*}{0,001} & \multirow{2}{*}{$0,000^{\mathrm{a}}$} \\
\hline & 36 a 50 & 4,213 & 0,4155 & & & & \\
\hline \multirow[t]{2}{*}{ Nasión } & 24 a 35 & 5,500 & 0,3071 & \multirow{2}{*}{4,000} & \multirow[b]{2}{*}{$-2,949$} & \multirow[b]{2}{*}{0,003} & \multirow{2}{*}{$0,002^{\mathrm{a}}$} \\
\hline & 36 a 50 & 3,675 & 1,0236 & & & & \\
\hline \multirow[t]{2}{*}{ A de Downs } & 24 a 35 & 8,625 & 0,3196 & \multirow{2}{*}{1,500} & \multirow{2}{*}{3,213} & \multirow{2}{*}{0,001} & \multirow{2}{*}{$0,000^{\mathrm{a}}$} \\
\hline & 36 a 50 & 7,013 & 0,8043 & & & & \\
\hline B de Downs & 24 a 35 & 9,600 & 0,2000 & & & & \\
\hline & 36 a 50 & 7,413 & 0,8643 & 2,500 & $-3,114$ & 0,002 & $0,001^{\mathrm{a}}$ \\
\hline Pogonion & 24 a 35 & 11,000 & 0,5806 & & & & \\
\hline & 36 a 50 & 8,725 & 0,6431 & 0,000 & $-3,336$ & 0,001 & $0,000^{\mathrm{a}}$ \\
\hline Gnathion & 24 a 35 & 6,238 & 0,1506 & & & & \\
\hline & 36 a 50 & 8,025 & 1,0846 & 8,000 & $-2,530$ & 0,011 & $0,001^{\mathrm{a}}$ \\
\hline S uperciliar izquierdo & 24 a 35 & 5,613 & 0,2357 & & & & \\
\hline & 36 a 50 & 5,525 & 0,3615 & 25,000 & $-0,743$ & 0,458 & $0,505^{\mathrm{a}}$ \\
\hline Superciliar derecho & 24 a 35 & 5,88 & 0,3227 & & & & \\
\hline & 36 a 50 & 5,913 & 0,5617 & 18,500 & $-1,439$ & 0,150 & $0,161^{*}$ \\
\hline Supraorbitario izquierdo & 24 a 35 & 5,663 & 0,3335 & & & & \\
\hline & 36 a 50 & 6,575 & 0,4621 & 6,000 & $-2,751$ & 0,006 & $0,005^{\mathrm{a}}$ \\
\hline S upraorbitario derecho & 24 a 35 & 5,738 & 0,4406 & & & & \\
\hline & 36 a 50 & 6,600 & 0,2204 & 1,500 & $-3,220$ & 0,001 & $0,000^{\mathrm{a}}$ \\
\hline Exocanthion izquierdo & 24 a 35 & 3,713 & 0,2167 & & & & \\
\hline & 36 a 50 & 4,163 & 0,2446 & 6,000 & $-2, / 57$ & 0,006 & $0,005^{\mathrm{a}}$ \\
\hline Exocanthion derecho & 24 a 35 & 3,788 & 0,3091 & & & & \\
\hline & 36 a 50 & 4,225 & 0,4234 & 12,500 & $-2,060$ & 0,039 & $0,038^{\mathrm{a}}$ \\
\hline Infraorbitario izquierdo & 24 a 35 & 3,838 & 0,6523 & & & & \\
\hline & 36 a 50 & 6,063 & 0,5041 & 0,000 & $-3,368$ & 0,001 & $0,000^{\prime \prime}$ \\
\hline Infraorbitario derecho & 24 a 35 & 3,925 & 0,6159 & & & & \\
\hline & 36 a 50 & 6,050 & 0,6279 & 0,000 & $-3,376$ & 0,001 & $0,000^{\mathrm{a}}$ \\
\hline Zygion izquierdo & 24 a 35 & 5,263 & 0,2264 & & & & \\
\hline & 36 a 50 & 6,813 & 0,2900 & 0,000 & $-3,393$ & 0,001 & $0,000^{\mathrm{a}}$ \\
\hline Zygion derecho & 24 a 35 & 5,275 & 0,1669 & & & & \\
\hline & 36 a 50 & 6,713 & 0,4324 & 0,000 & $-3,3 / 8$ & 0,001 & $0,000^{2}$ \\
\hline Gonion izquierdo & 24 a 35 & 17,438 & 0,4689 & & & & \\
\hline & 36 a 50 & 9,163 & 0,7782 & 0,000 & $-3,368$ & 0,001 & $0,000^{\mathrm{a}}$ \\
\hline Gonion derecho & 24 a 35 & 17,463 & 0,4749 & & & & \\
\hline & 36 a 50 & 9,238 & 0,8070 & 0,000 & $-3,381$ & 0,001 & $0,000^{a}$ \\
\hline
\end{tabular}

a. No corregidos para los empates. b. Variable de agrupación. 
Tabla IV. Valores de grosor tisular medido en grupo entre 24 y 35 años y correlación por sexo.

\begin{tabular}{|c|c|c|c|c|c|c|c|}
\hline Punto & Sexo & Promedio & $\begin{array}{c}\text { Desviación } \\
\text { estándar }\end{array}$ & $\begin{array}{l}\text { U de Mann- } \\
\text { Whitney }\end{array}$ & Z & $\begin{array}{l}\text { Sig. asintót ica } \\
\text { (bi lateral) }\end{array}$ & $\begin{array}{l}\text { Sig. exacta } \\
\text { (unilateral) }\end{array}$ \\
\hline \multirow[t]{2}{*}{ Trichion } & Masculino & 5,488 & 0,1885 & 0,000 & $-3,368$ & 0,001 & $0,000^{\mathrm{a}}$ \\
\hline & Femenino & 3,525 & 0,2816 & & & & \\
\hline \multirow[t]{2}{*}{ Supraglabela } & Masculino & 4,675 & 0,1282 & 0,000 & $-3,376$ & 0,001 & $0,000^{\mathrm{a}}$ \\
\hline & Femenino & 3,475 & 0,3012 & & & & \\
\hline \multirow[t]{2}{*}{ Glabela } & Masculino & 5,550 & 0,1604 & 26,500 & 0,0586 & 0,558 & $0,574^{\mathrm{a}}$ \\
\hline & Femenino & 5,650 & 0,2507 & & & & \\
\hline \multirow[t]{2}{*}{ Nasion } & Masculino & 5,988 & 0,1458 & 4,000 & $-2,956$ & 0,003 & $0,002^{\mathrm{a}}$ \\
\hline & Femenino & 5,500 & 0,3071 & & & & \\
\hline \multirow[t]{2}{*}{ A de Downs } & Masculino & 11,250 & $0,13.09$ & 0,000 & $-3,383$ & 0,001 & $0,000^{\mathrm{a}}$ \\
\hline & Femenino & 8,625 & 0,3196 & & & & \\
\hline \multirow[t]{2}{*}{ B de Downs } & Masculino & 10,238 & 0,1408 & 0,000 & $-3,371$ & 0,001 & $0,000^{\mathrm{a}}$ \\
\hline & Femenino & 9,600 & 0,2000 & & & & \\
\hline \multirow[t]{2}{*}{ Pogonion } & Masculino & 11,675 & 0,1035 & 15,000 & $-1,799$ & $.0,72$ & $0,830^{\mathrm{a}}$ \\
\hline & Femenino & 11,000 & 0,5806 & & & & \\
\hline \multirow[t]{2}{*}{ Gnathion } & Masculino & 6,675 & 0,0886 & 0,000 & $-3,403$ & 0,001 & $0,000^{\mathrm{a}}$ \\
\hline & Femenino & 6,238 & 0,1506 & & & & \\
\hline \multirow[t]{2}{*}{ S uperciliar izquierdo } & Masculino & 5,838 & 0,1685 & 11,500 & $-2,195$ & 0,028 & $0,028^{\mathrm{a}}$ \\
\hline & Femenino & 5,613 & 0,2357 & & & & \\
\hline \multirow[t]{2}{*}{ Superciliar derecho } & Masculino & 5,775 & 0,1389 & 22,000 & $-1,064$ & 0,287 & $0,328^{\mathrm{a}}$ \\
\hline & Femenino & 5,588 & 0,3227 & & & & \\
\hline \multirow[t]{2}{*}{ Supraorbitario izquierdo } & Masculino & 5,738 & 0,0744 & 27,500 & $-0,483$ & 0,629 & $0,645^{\mathrm{a}}$ \\
\hline & Femenino & 5,663 & 0,3335 & & & & \\
\hline \multirow[t]{2}{*}{ Supraorbitario derecho } & Masculino & 5,875 & 0,0886 & 28,000 & $-0,432$ & 0,665 & $0,721^{\mathrm{a}}$ \\
\hline & Femenino & 5,738 & 0,4406 & & & & \\
\hline \multirow[t]{2}{*}{ Exocanthion izquierdo } & Masculino & 3,725 & 0,1488 & 31,500 &,- 054 & 3,957 & $0,959^{\mathrm{a}}$ \\
\hline & Femenino & 3,713 & 0,2167 & & & & \\
\hline \multirow[t]{2}{*}{ Exocanthion derecho } & Masculino & 3,763 & 0,1923 & 29,500 & $-0,265$ & 0,791 & $0,798^{\mathrm{a}}$ \\
\hline & Femenino & 3,788 & 0,3091 & & & & \\
\hline \multirow[t]{2}{*}{ Infraorbitario izquierdo } & Masculino & 4,400 & 0,1773 & 8,000 & $-2,534$ & 0,011 & $0,010^{a}$ \\
\hline & Femenino & 3,838 & 0,6523 & & & & \\
\hline \multirow[t]{2}{*}{ Infraorbitario derecho } & Masculino & 4,400 & 0,2673 & 16,000 & $-1,689$ & 0,091 & $0,105^{\mathrm{a}}$ \\
\hline & Femenino & 3,925 & 0,6159 & & & & \\
\hline \multirow[t]{2}{*}{ Zygion izquierdo } & Masculino & 5,000 & 0,2000 & 13,000 & $-2,021$ & 0,043 & $0,050^{\mathrm{a}}$ \\
\hline & Femenino & 5,263 & 0,2264 & & & & \\
\hline \multirow[t]{2}{*}{ Zygion derecho } & Masculino & 4,988 & 0,2167 & 7,000 & $-2,706$ & 0,007 & $0,007^{\mathrm{a}}$ \\
\hline & Femenino & 5,275 & 0,1669 & & & & \\
\hline \multirow[t]{2}{*}{ Gonion izquierdo } & Masculino & 5,163 & 0,1061 & 0,000 & $-3,381$ & 0,001 & $0,000^{\mathrm{a}}$ \\
\hline & Femenino & 17,438 & 0,4689 & & & & \\
\hline \multirow[t]{2}{*}{ Gonion derecho } & Masculino & 5,125 & 0,1581 & 0,000 & $-3,406$ & 0,001 & $0,000^{\mathrm{a}}$ \\
\hline & Femenino & 17,463 & 0,4749 & & & & \\
\hline
\end{tabular}

a. No corregidos para los empates. b. Variable de agrupación. 
Tabla V. Valores de grosor tisular medido en grupos entre 36 y 50 años y correlación por sexo.

\begin{tabular}{|c|c|c|c|c|c|c|c|}
\hline Puntos & Sexo & Promedio & Devesta & $\begin{array}{c}\text { U de Mann- } \\
\text { Witney }\end{array}$ & Z & $\begin{array}{l}\text { Sig, asintótica } \\
\text { (bilateral) }\end{array}$ & $\begin{array}{l}\text { Sig, exacta } \\
\text { (unilateral) }\end{array}$ \\
\hline \multirow[t]{2}{*}{ Trichion } & Masculino & 4,750 & 0,5014 & 26,000 & $-0,634$ & 0,526 & $0,574^{\mathrm{a}}$ \\
\hline & Femenino & 4,775 & 0,3845 & & & & \\
\hline \multirow[t]{2}{*}{ Supraglabela } & Masculino & 4,425 & 0,5175 & 9,500 & $-2,391$ & 0,017 & $0,015^{\mathrm{a}}$ \\
\hline & Femenino & 3,875 & 0,2375 & & & & \\
\hline \multirow[t]{2}{*}{ Glabela } & Masculino & 5,325 & 0,8515 & 4,000 & $-2,949$ & 0,003 & $0,002^{\mathrm{a}}$ \\
\hline & Femenino & 4,213 & 0,4155 & & & & \\
\hline \multirow[t]{2}{*}{ Nasión } & Masculino & 6,113 & 0,4518 & 1,000 & $-3,263$ & 0,001 & $0,000^{\mathrm{a}}$ \\
\hline & Femenino & 3,675 & 1,0236 & & & & \\
\hline \multirow[t]{2}{*}{ A de Downs } & Masculino & 11,950 & 0,9040 & 0,000 & $-3,363$ & 0,001 & $0,000^{\mathrm{a}}$ \\
\hline & Femenino & 7,013 & 0,8043 & & & & \\
\hline \multirow[t]{2}{*}{ B de Downs } & Masculino & 11,200 & 0,3338 & 0,000 & $-3,378$ & 0,001 & $0,000^{\mathrm{a}}$ \\
\hline & Femenino & 7,413 & 0,8643 & & & & \\
\hline \multirow[t]{2}{*}{ Pogonion } & Masculino & 11,050 & 0,6188 & 1,000 & $-3,260$ & 0,001 & $0,000^{\mathrm{a}}$ \\
\hline & Femenino & 8,725 & 0,6431 & & & & \\
\hline \multirow[t]{2}{*}{ Gnathion } & Masculino & 8,938 & 1,5583 & 25,500 & $-0,683$ & 0,495 & $0,505^{\mathrm{a}}$ \\
\hline & Femenino & 8,025 & 1,0846 & & & & \\
\hline \multirow[t]{2}{*}{ S uperciliar izquierdo } & Masculino & 5,788 & 0,7396 & 14,000 & $-1,906$ & 0,057 & $0,065^{\mathrm{a}}$ \\
\hline & Femenino & 5,525 & 0,3615 & & & & \\
\hline \multirow[t]{2}{*}{ Superciliar derecho } & Masculino & 5,850 & 0,5210 & 30,000 & $-0,220$ & 0,826 & $0,878^{\mathrm{a}}$ \\
\hline & Femenino & 5,913 & 0,5617 & & & & \\
\hline \multirow[t]{2}{*}{ S upraorbitario izquierdo } & Masculino & 7,975 & 1,0607 & 7,000 & $-2,639$ & 0,008 & $0,007^{\mathrm{a}}$ \\
\hline & Femenino & 6,575 & 0,4621 & & & & \\
\hline \multirow[t]{2}{*}{ Supraorbitario derecho } & Masculino & 8,288 & 0,5167 & 0,000 & $-3,381$ & 0,001 & $0,000^{\mathrm{a}}$ \\
\hline & Femenino & 6,600 & 0,2204 & & & & \\
\hline \multirow[t]{2}{*}{ Exocanthion izquierdo } & Masculino & 4,200 & 0,5318 & 23,500 & $-0,903$ & 0,366 & $0,382^{\mathrm{a}}$ \\
\hline & Femenino & 4,163 & 0,2446 & & & & \\
\hline \multirow[t]{2}{*}{ Exocanthion derecho } & Masculino & 4,238 & 0,4596 & 28,500 & $-0,371$ & 0,711 & $0,721^{\mathrm{a}}$ \\
\hline & Femenino & 4,225 & 0,4234 & & & & \\
\hline \multirow[t]{2}{*}{ Infraorbitario izquierdo } & Masculino & 7,213 & 0,7643 & 4,000 & $-2,958$ & 0,003 & $0,002^{\mathrm{a}}$ \\
\hline & Femenino & 6,063 & 0,5041 & & & & \\
\hline \multirow[t]{2}{*}{ Infraorbitario derecho } & Masculino & 7,088 & 0,5139 & 6,500 & $-2,704$ & & $0,005^{\mathrm{a}}$ \\
\hline & Femenino & 6,050 & 0,6279 & & & 0,007 & \\
\hline \multirow[t]{2}{*}{ Zygion izquierdo } & Masculino & 10,013 & 2,4701 & 8,000 & $-2,534$ & 0,011 & $0,010^{\mathrm{a}}$ \\
\hline & Femenino & 6,813 & 0,2900 & & & & \\
\hline \multirow[t]{2}{*}{ Zygion derecho } & Masculino & 10,225 & 1,9962 & 8,000 & $-2,530$ & 0,011 & $0,010^{\mathrm{a}}$ \\
\hline & Femenino & 6,713 & 0,4324 & & & & \\
\hline \multirow[t]{2}{*}{ Gonion izquierdo } & Masculino & 9,850 & 2,0935 & 13,000 & $-1,998$ & 0,046 & $0,050^{\mathrm{a}}$ \\
\hline & Femenino & 9,163 & 0,7782 & & & & \\
\hline \multirow[t]{2}{*}{ Gonion derecho } & Masculino & 9,750 & 1,9228 & 15,500 & $-1,744$ & 0,081 & $0,083^{\mathrm{a}}$ \\
\hline & Femenino & 9,238 & 0,8070 & & & & \\
\hline
\end{tabular}

a. No corregidos para los empates. b. Variable de agrupación. c. Grupo etáreo $=36$ - 50 años. 
El presente estudio establece variaciones en el grosor del tejido blando a través del tiempo. Al correlacionar los valores de medición con la edad de los cadáveres estudiados, en los varones se observó un aumento estadísticamente significativo en el grosor tisular tanto en la región mediana como en la región paramediana del rostro. Esta tendencia no fue observada tan marcadamente en las mujeres, a diferencia de lo planteado por Stephan et al. (2005) quienes sostienen que existe un incremento en el grosor tisular para este género a través del tiempo. En contraste con lo citado anteriormente, la correlación demuestra que las mujeres tienden a igualar en el grosor tisular, a los varones en el tiempo, situación que es insinuada en el adulto joven, y establecida en la vejez (Domaracki \& Stephan).

Los resultados de este estudio se suman a los ya descritos por otras investigaciones (De Greef et al., 2006), permitiendo enriquecer y actualizar la información existente sobre la profundidad del tejido blando craneofacial, además de proporcionar una mejor aproximación de los valores de medición de la población en estudio; todo lo anterior en beneficio del campo de la reconstrucción facial por escultura forense aplicada a la identificación médicolegal.

SUAZO, G. I. C., PÉREZ, R. F. J. \& TORRES, M. S. R. Tissue tickness in spanish corpses and your applications on the medicolegal identification. Int. J. Morphol., 25(1):109-116, 2007.

SUMMARY:The facial reconstruction for forensic sculpture is a skill in which, by means of the shaped one with plastic materials on a cephalic skeleton, one reproduces soft parts of the face with the intention of serving to the process of medicolegal identification. One of the most relevant information to reproduce this skill are the values of thickness tisular in certain points of the skeleton craneal. In the present study the thickness tissues are analyzed in 14 craneometrics points median and paramedian of 32 Spanish corpses by means of the introduction of needles from the facial skin. The corpses were divided in groups of agreement to sex and age to that they belong.

They found significant differences in the values of thickness tisular between men and women of both groups etáreos, being observed that the men a major thickness tisular in the median points, whereas in the women the thickness measured in points turned out to be major paramedian.

KEY WORDS: Skinfold thickness; Forensic antropology, Human identification.

\section{REFERENCIAS BIBLIOGRÁFICAS}

De Greef, S.; Claes, P.; Vandermeulen, D.; Mollemans, W.; Suetens, P. \& Willems, G. Large-scale in-vivo Caucasian facial soft tissue thickness database for craniofacial reconstruction. Forensic. Sci. Int., 159:26-146, 2006.

Domaracki, M. \& Stephan, C. Facial Soft tissue thicknesses in Australian Adult Cadavers. J. Forensic., SCI, 51(1):5-10, 2006.

Dumont, E. R. Mid-facial tissue depths of white children: an aid in facial feature reconstruction. J. Forensic. SCI., 31:1463-9, 1986.

El-Mehallawi, I. H. \& Soliman, E. M. Ultrasonic assessment of facial soft tissue thicknesses in adult Egyptians. Forensic., Sci. Int., $117(1-2): 99-107,2001$.

Farkas, L. G. Anthropometry of the head and face. New York, Raven Press, 1994.

Manhein, M. H.; Listi, G. A.; Barsley, R. E.; Musselman, R.; Barrow, N. E. \& Ubelaker, D. H. In vivo facial tissue delht measurements for children and adults. J. Forensic. Sci., 45:4860, 2000.

Rhine, J. S. \& Campbell, H. R. Thickness of facial tissues in American blacks. J. Forensic. SCI., 25:847-58, 1980.
Rodríguez, J. V. La Antropología Forense en la Identificación Humana. Bogotá, Anaconda, 2003.

Simpson, E. \& Henneberg, M. Variation in soft-tissue thicknesses on the human face and their relation to craniometric dimensions. Am. J. Phys. Anthropol., 118:121-33, 2002.

Stephan, C. N.; Norris, R. M. \& Henneberg, M. Does sexual dimorphism in facial soft tissue depths justify sex distintion in craniofacial identification?. J. Forensic. SCI., 50:513-8, 2005.

Teke, A. Medicina Legal y Criminalística, Santiago de Chile, Editorial Jurídica de Santiago, 2004.

Wilkinson, C. M. In vivo facial tissue depth measurements for White British children. J. Forensic. Sci., 47(3):459-65, 2002.

Dirección para correspondencia:

Dr. Iván Claudio Suazo Galdames

Unidad de Anatomía Normal

Universidad de Talca

Avenida Lircay s/n Oficina N462

Talca - CHILE

Recibido : 24-10-2006

Email: isuazo@utalca.cl 